\title{
Upstream Filtration Preparation of Poor Sections of Deposits before Development
}

\author{
Alexandr Gennadievich Mikhailov, Ivan Ivanovich Vashlaev, Margarita Yurievna Kharitonova \\ Institute of Chemistry \& Chemical Technology, Federal Research Center Krasnoyarsk Science Center, Siberian Branch of the \\ Russian Academy of Sciences (ICCT SB RAS), Krasnoyarsk, Russia \\ Email: mag@icct.ru
}

How to cite this paper: Mikhailov, A.G., Vashlaev, I.I. and Kharitonova, M.Yu. (2018) Upstream Filtration Preparation of Poor Sections of Deposits before Development. Journal of Minerals and Materials Characterization and Engineering, 6, 395-407.

https://doi.org/10.4236/jmmce.2018.63028

Received: April 13, 2018

Accepted: May 18, 2018

Published: May 21, 2018

Copyright ( $) 2018$ by authors and Scientific Research Publishing Inc. This work is licensed under the Creative Commons Attribution International License (CC BY 4.0).

http://creativecommons.org/licenses/by/4.0/

\begin{abstract}
Forming a new concentrated sense of useful components in conditions of nature, geological processes allows us to create techogenous deposits of required quality and forms in situ. The article presents a variant of the preparation of the deposit by a filtration geological process in situ. Structural and material transformation of the massif of hypergenesis nature methods allows bringing qualitative indicators to the required condition values. This will ensure the effective development of the deposit with traditional technological solutions. Experimental results of aqueous filtration are described. The schemes of technological solutions for natural and technogenic deposits are considered.
\end{abstract}

\section{Keywords}

Capillary Rise, Groundwater, Dissolution, Sedimentation, Concentration, Aeration Zone

\section{Introduction}

The efficiency of the development of a part of the deposit area depends to a large extent on the technology used. The technology determines the mineral reserves and conditions of development as a useful component and can significantly change the availability of a deposit. The application of a specific technological base is crucial. Exploitation of the deposits in the aggregate of the mineral and raw material base follows from the patterns of development of the mining technology complex. The forecast of the field includes sequence depends on the basis of the technological level by g/t diagram [1]. The main factors determining the availability index are the main conditions, such as the aggregate volume of reserves, the average and the content in the air, the depth of deposition (the coefficient of stripping), and so on. In addition, the most important indicators include 
the infrastructure development of the territory and climatic conditions. This fully characterizes the access to the full range of mineral resources, both within each species, and in general for the mineral resource base. This approach is also applicable to technogenic objects. Among the illustrative examples, one can use a vivid example of the application of technologies for heap leaching of gold in mineral-raw gold mining. The technology has been reduced to the requirements for gold content in deposit. In a short time, a large number of poor deposits were involved in the development. As a result, a large-scale increase in production based on this technology provided a strategic advantage to states (USA, Australia).

Technological features allow such fields to pass into the category of available deposits to the applicable technological conditions and will be worked out profitable. There is no doubt that the technological development on the one hand and the laws of market relations on the other, will allow to correct the conditions and involve poorer and more difficult raw materials, influencing the price position. One of the tools of technological influence is the direction of field preparation in terms of the composition and structure of the components in the massif (in situ) [2]. This is a fairly new view on the potential of mineral raw materials. The process of direct material and structural transformation of the massif in order to bring the indicators to the conditioning values will allow the effective development of reserves in accordance with existing technological approaches by changing the parameters of the quantity and quality of the useful component in situ. The prospect of this approach ensures the expansion of the mineral and raw material base due to the substandard sections of existing deposits, ore occurrences (subsoil plots that did not become deposits as required by the standards) and man-made objects. The basis for this direction of the preparation of the massifs is the well-known geological regularities in the formation of deposits [3], and studies of the mineral transformation of technogenic massifs in hypergenesis [secondary mineral formation].

Among the totality of natural types of deposits, a significant role is played by infiltration deposits, the formation of which is caused by the filtration redeposition of useful components. Instruments in the formation of zones of secondary concentration are the patterns of movement of mineralized fluids in the massif and sedimentation of useful components of a chemical and physical barrier. The application of patterns of direct redeposition will determine the construction of the basis for a directed physical-chemical transformation in the zone of secondary mineral formation. The intensity of mineral transformations and the concentration of useful components in technogenic objects, in many cases, are many times higher than the primary concentration [3]. It's connected, most likely, with a higher active potential between solutions and barriers of deposition. The variant of the vertical capillary rise of solutions in the near-surface zone of the array-the zone of aeration-is quite promising for using technology. The movement of fluids up to the surface is based on the mechanism of capillary 
upward of the liquid and is associated, mainly, with the magnitude of atmospheric humidity. The latter is the motivating factor of the direction of movement. Water evaporates from the surface and in the aeration zone and "frees up" the space for newly introduced portions of the solution. The difference in humidity in the atmosphere and in the aeration zone serves as a "pump" (pressure gradient) that lifts the solution to the surface. The motion of solutions in the aeration zone is well represented in the field of soil science [4]. In the conditions of hypergenesis, the main substance converting, in fact, factor is the oxidation process. Delivery of oxygen to the massif can be carried out both through aeration from the atmosphere, and groundwater [3].

In the hypergenesis zone, almost all the elements can be represented by different phase states [5]. Their presence in exchange phase, carbonate and organic phase states are water-soluble and can migrate to filtration waters. In addition, water filtration will cause the delivery of oxygen to the massif, which will support the oxidation (hypergene) process in the massif. The applicability of water as a reagent for the real directional transformation of a hypergene massif with direct filtration ascending motion of solutions can be evaluated by the results of experimental studies. The mechanism of ascending capillary ascent is new and is not used in technology [5] [6]. Also, the possibility of using an aqueous base for performing the filtration mass transfer has not been sufficiently studied. The process of formation of concentration zones directed to the surface can also be used to prepare high concentrations of useful components the near-surface areas of the massif [7]. A number of experimental studies will allow us to give a preliminary assessment of possible directed real transformations.

\section{Materials and Methods}

The object of experimental research was the material of tails of flotation enrichment of sulfide copper-nickel ores. This material is a waste of the mining and metallurgical plant in the Norilsk industrial area. It was used to carry out experimental studies on the mass transfer of water-soluble phase. The sample is mainly represented by rock-forming minerals-aluminosilicates (muscovite, elite, serpentine, acagenite) and quartz. This was established by X-ray phase analysis. Ore minerals are represented by pyrrhotite, chromite, subordinated by chalcopyrite, gypsum, rarely calcite, brucite, pentlandite. The content of sulfide minerals reaches $10 \%$. In appearance, the tailings of enrichment are gray sand, the main fraction lies in the interval $0.1-0 \mathrm{~mm}$ (Table 1 ). The density of the rock particles is $2.42 \mathrm{t} / \mathrm{m}^{3}$, the bulk density is $1.47 \mathrm{t} / \mathrm{m}^{3}$. The granulometric composition of the material is presented in Table 1.

To study the nature of the motion of solutions and the precipitation of water-soluble salts, a calculation procedure was used for the rate of filtration of mineralized solutions in loose masses. The technique is calculated for the conditions of the gradient supply of solutions of the section of the massif.

Experimental setups for the ascending rise of solutions in the massif and the 
study of the vertical zoning of the precipitation of secondary minerals in the aeration zone of the massif were developed and manufactured. The principle of the Mariotta vessel was used to maintain a constant level of water table of solution in the massif. It was adapted to the conditions of the experimental setups. Installations are simulating a massif of containers in various shapes (a cylinder made of polycarbonate with a diameter of $110 \mathrm{~mm}$, an inverted pyramid, an oblique column of rectangular cross section) filled with ore material from waste enrichment. The height of the tanks is $1.5 \mathrm{~m}$. The flow of solutions is organized from the bottom of each tank (Figure 1). In the each tanks the ore material of the tailings of enrichment was used only the classified material in a narrow range. $\mathrm{A}$ series of experiments was started to reveal the regularities of precipitation and accumulation of salts under phase transition conditions when moving through capillaries.

Formation of concentration zones in the near-surface aeration zone of the massif with the use of an evaporation barrier (and without a barrier) was experimentally

Table 1. Granulometric composition of the sample.

\begin{tabular}{cccc}
\hline \multirow{2}{*}{ size, $\mathrm{mm}$} & average size, $\mathrm{mm}$ & $\mathrm{g}$ & number, \\
\cline { 3 - 4 } & & $\mathrm{g}$ & $\%$ \\
\hline$-5+1$ & 1.5 & 18.3 & 1.67 \\
$-1+0.5$ & 0.75 & 69 & 6.28 \\
$-0.5+0.2$ & 0.35 & 113.5 & 10.34 \\
$-0.2+0.125$ & 0.1625 & 125.3 & 11.41 \\
$-0.125+0.1$ & 0.1125 & 221.8 & 20.20 \\
$-0.1+0.063$ & 0.0815 & 170.8 & 15.56 \\
-0.063 & 0.0315 & 379.3 & 34.54 \\
total & & 1098 & 100 \\
\hline
\end{tabular}

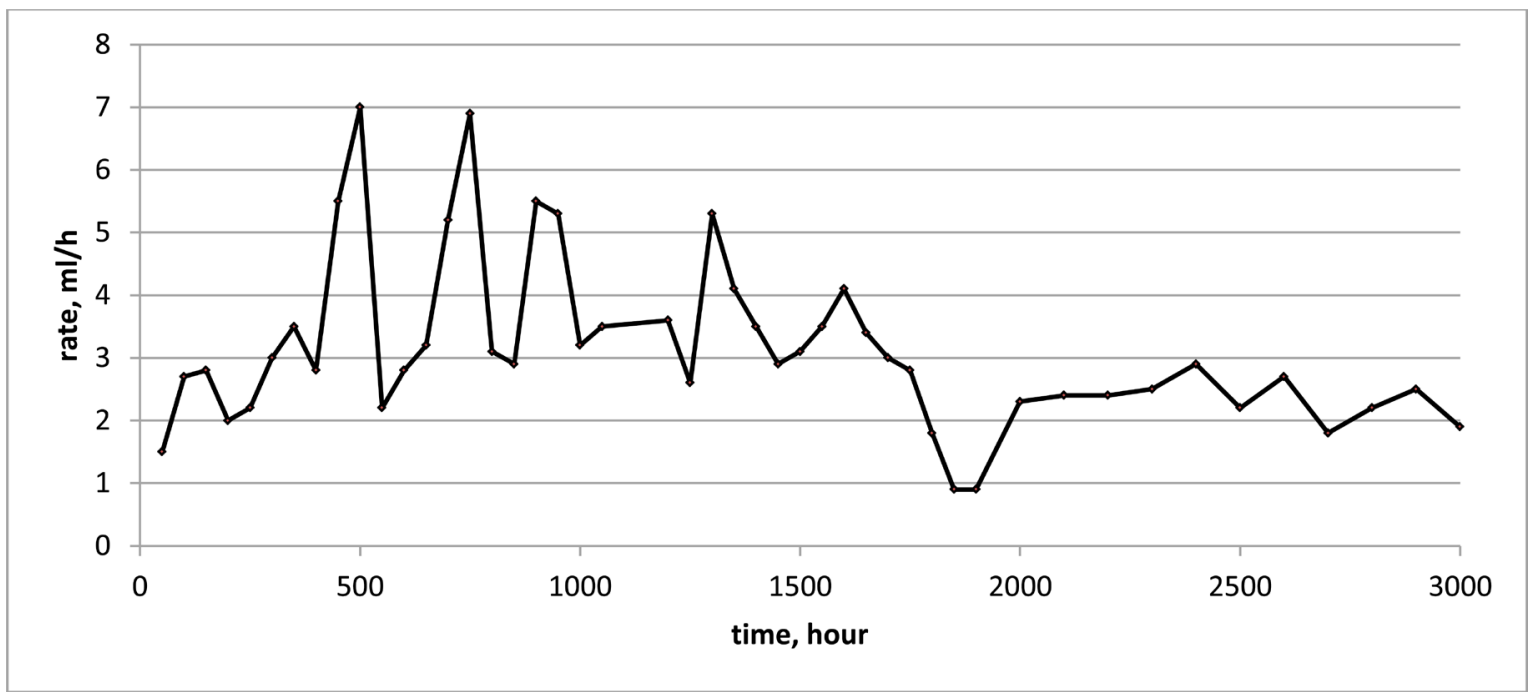

Figure 1. The rate of the filtration upward flow of the aqueous solution. 
carried out at different vertical lifting installations of solutions [7] [8]. In the simulated array, water was supplied as an active reagent for transferring water-soluble compounds of non-ferrous metals in solution. In the course of the experiment, the change in the filtration rate for various forms, structures, capillary sizes, and changes in the material composition was studied. The duration of the experiments was different from several days and months to six months, and even up to 2 years (assessment of the filtration ability of the mineralized solution). The distribution of elements by geochemical phase states was carried out by the method of geochemical analysis [5]. The productivity of the consists solutions as they were raised vertically through the capillaries of the massif was estimated by atomic-adsorption analysis. At different heights of the tanks, access to sampling of both solutions and solid material is established (Figure 1).

\section{Results and Discussions}

During the experiment, especially in the initial period, the fluctuations in the filtration flow rate are unstable. The fluctuations are $2-4$ times relative to the average value (Figure 2). The feed level corresponds to the surface level. The calculated velocity of the filtration flow in the initial period is $1 / 2$ of the experimental value.

The filtration instability of the flow of fluids through the capillaries with such fluctuations from the average calculated value can be justified by periodic localization from uniform motion throughout the volume to focusing by separate channels. In the water-soluble solutions, they begin to react with its mineral components and establish channels in the rock that are many times larger than the size of the capillaries. As the solution moves through the capillaries due to the action of reactive infiltration.

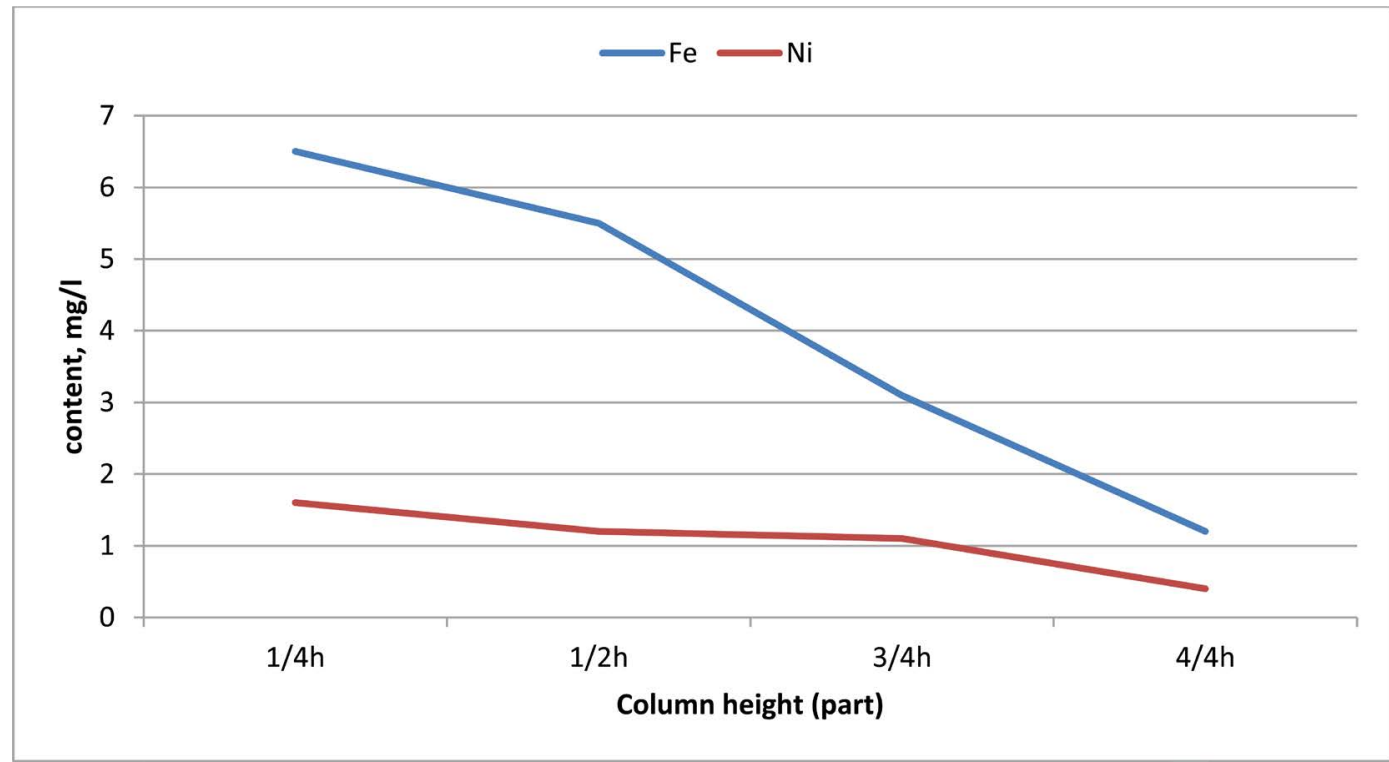

Figure 2. Change in the content of iron and nickel in solution over the height of the column at the beginning of the experiment 
Instability, the oscillations have multiple changes associated with increased porosity of the sections. This causes a local increase in the velocity of the fluids. This leads to a more rapid local increase in permeability and, in turn, causes acceleration of the reaction front motion. The neighboring channels massif is excluded from the process. The reaction front decays when the activity of the solution or the mineral components of the massif is reduced. In addition, simultaneously during the movement of solutions along the formed channels, including the capillaries, the partially mineral phase precipitates from the liquid to the solid phase, and forms deposits, not excluding the complete overlap of the live section of the capillaries of the individual sections. The presence of the colmatation effect is indicated by the data of the episodic decrease in the velocity of the solutions in the massif and the change in mineralization of solutions at different horizons of the massif, and in different time. The average decrease in the rate of capillary flow of solutions, according to the experimental data, does not exceed $25 \%$ of the mean value, and, as a rule, appear immediately after the peak of the decrease (Figure 2).

The degree of transition into the solution of non-ferrous compounds of copper, cobalt and nickel is low. For $\mathrm{Cu}-$ up to $0.05 \%$, $\mathrm{Co}-$ up to $0.6 \%, \mathrm{Ni}-$ up to $0.11 \%$. A low level of concentration is maintained throughout the experiment 16 18 months. At the beginning and at the end of the experimental period, the character of the change in the concentrations of elements in the solution varies in levels. In the initial segment, the concentration decreases to the surface (Figure 2, Figure 3). In the final interval, the concentration of all elements in the solution rises to the surface. This is due to the step-by-step redeposition of the connections as the upward movement proceeds (Figure 4, Figure 5). At the beginning, the compounds precipitate out of the solution, to a greater extent, in the lower zone, but gradually the front of the deposition zone shifts to the surface.

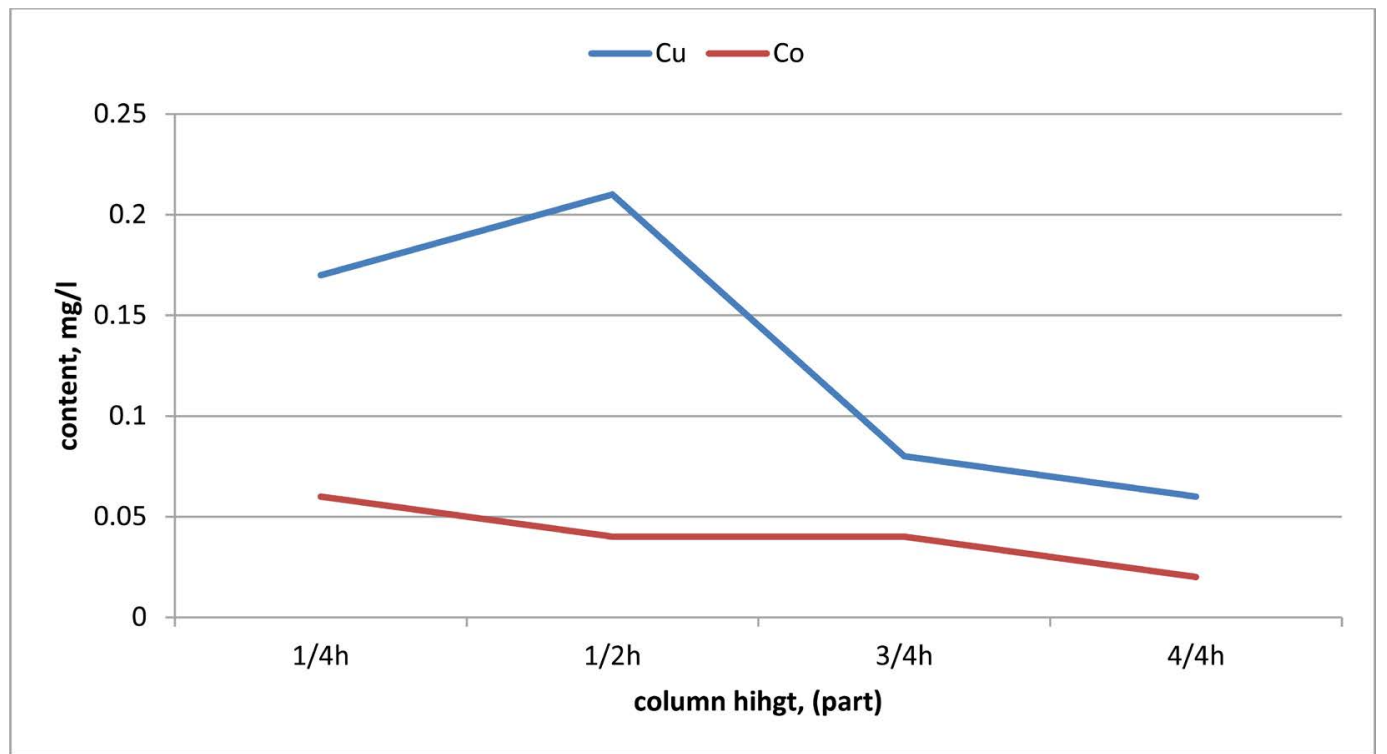

Figure 3. Change in the content of $\mathrm{Cu}, \mathrm{CO}$ in solution over the height of the column with capillary rise at the beginning of the experiment. 


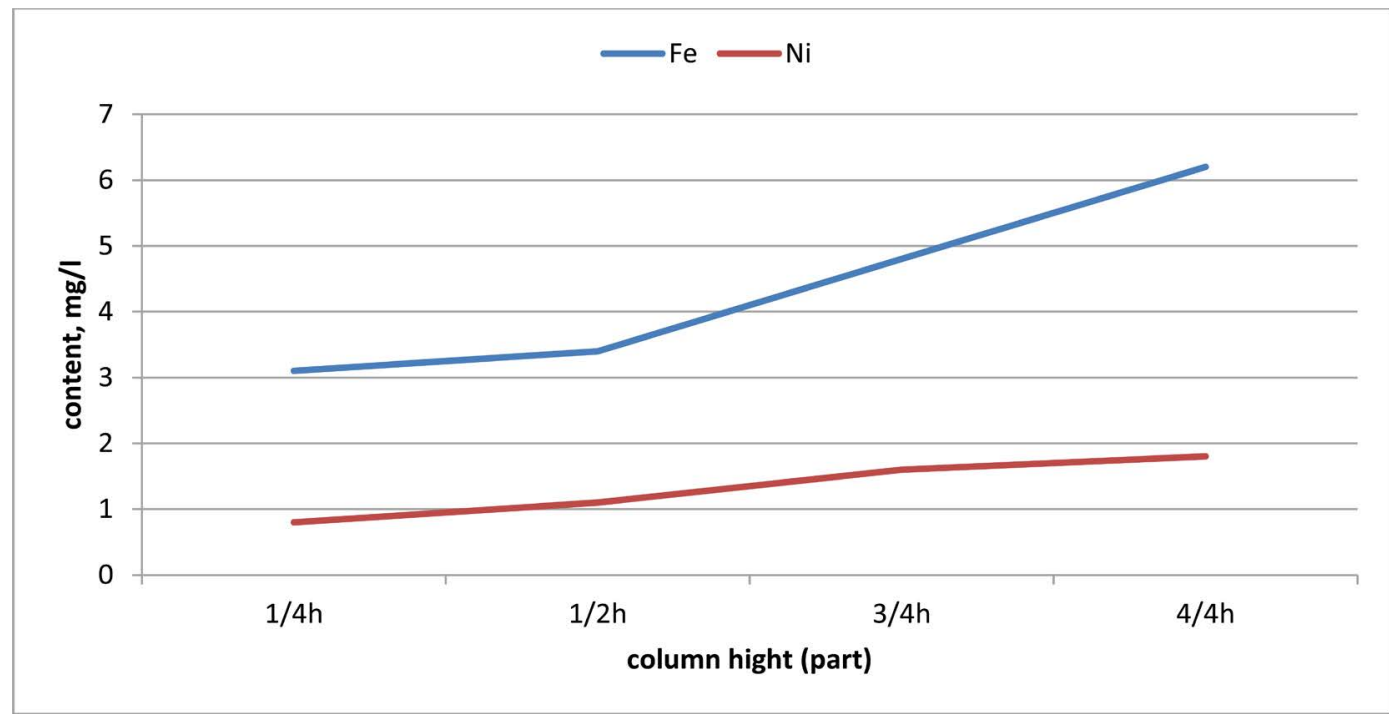

Figure 4. Change in the content of iron and nickel in solution by the column height at the end of the experiment.

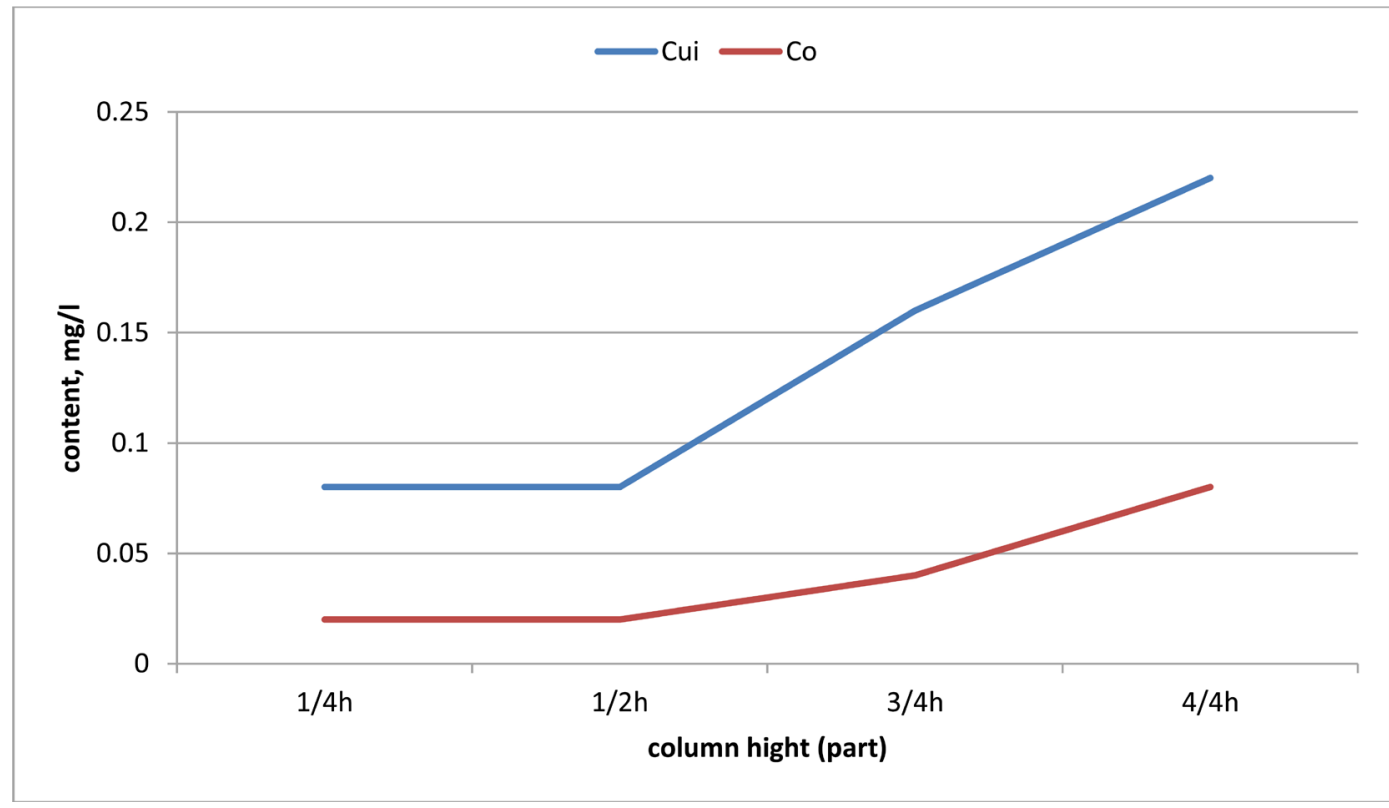

Figure 5. Change in content of $\mathrm{Cu}$, Co in solution by column height at capillary rise at the end of the experiment.

Basically, during this period of the experiment, practically the entire water-soluble part of non-ferrous metals are extracted, the share of which was estimated by the geochemical phase analysis prior to the onset of the experiment. At the end of the experiment, a repeated geochemical phase analysis was performed, which showed that the proportion of water-soluble phases of the elements of non-ferrous metals decreased, but did not disappear, despite the fact that during the experimental period the entire volume (according to calculations) of the exchange, carbonate and organic phases was recovered. Simultaneously, the share of crystalline oxides and sulfides of non-ferrous metals decreased. This, most 
likely, shows that the water-soluble forms of non-ferrous metals in the conditions of hypergenesis are replenished from crystalline oxides and sulfides, i.e. In the mass if the oxidation process proceeds. The dynamics of the mobilization of non-ferrous metals in the solution depends on the $\mathrm{pH}$ of the medium and increases in an acidic (in the extreme case, a weakly acidic) medium. Weak acidification of the supplied water to $\mathrm{pH} 5$ increased the concentration of non-ferrous metals in 2 to 2.5 times in the solution at the initial stage and, after 1.5 months, was compared with the concentrations of solutions in which acidification was not applied. This concentration was retained until to the end 6 months and then decreased sharply. Without artificial acidification of the feed water, a neutral medium was maintained during the entire experiment period, despite the expectation of the oxidation process of the sulfides present in the material of the array and the presence of dissolved oxygen in the supplied water. As a result of statistical analysis it was established that the contents of almost all non-ferrous metals have a close correlation with the content of ferrous iron in the solution. The coefficient of pair correlation between iron and each element $(\mathrm{Co}, \mathrm{Cu}, \mathrm{Ni})$ is in the range from 0.68 to 0.87 . The significant value of the correlation bonds allows us to assume that the content of the elements of non-ferrous metals depends indirectly on the presence of $\mathrm{Fe}+2$. The dynamics of the change in the $\mathrm{Fe}$ +2 content over the first two months in a solution taken from the surface of the column are shown in Figure 6.

The content of $\mathrm{Cu}, \mathrm{Co}, \mathrm{Ni}$ in the solution is low and increases by $\mathrm{Ni}$-almost twice to $1.9 \mathrm{mg} / \mathrm{l}$ by the end of the first month. The content of $\mathrm{Cu}$ and $\mathrm{Co}$ increases sharply towards the end of the second month and remains at these values almost to the end of the experiment. The dynamics of the contents are shown in Figure 7.

For all the observed elements-cobalt, nickel, copper, a pronounced dependence

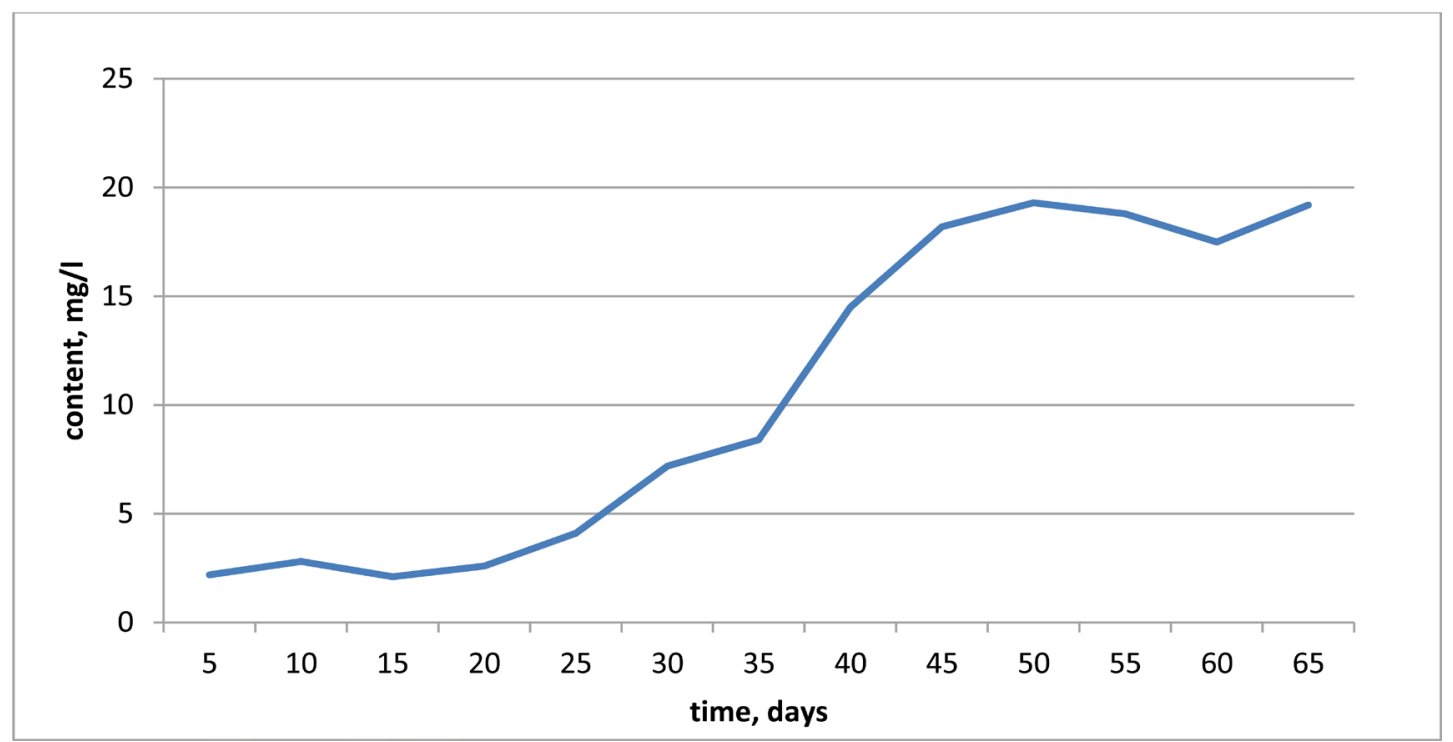

Figure 6. Fe content in solution on the surface of the material in the column. 


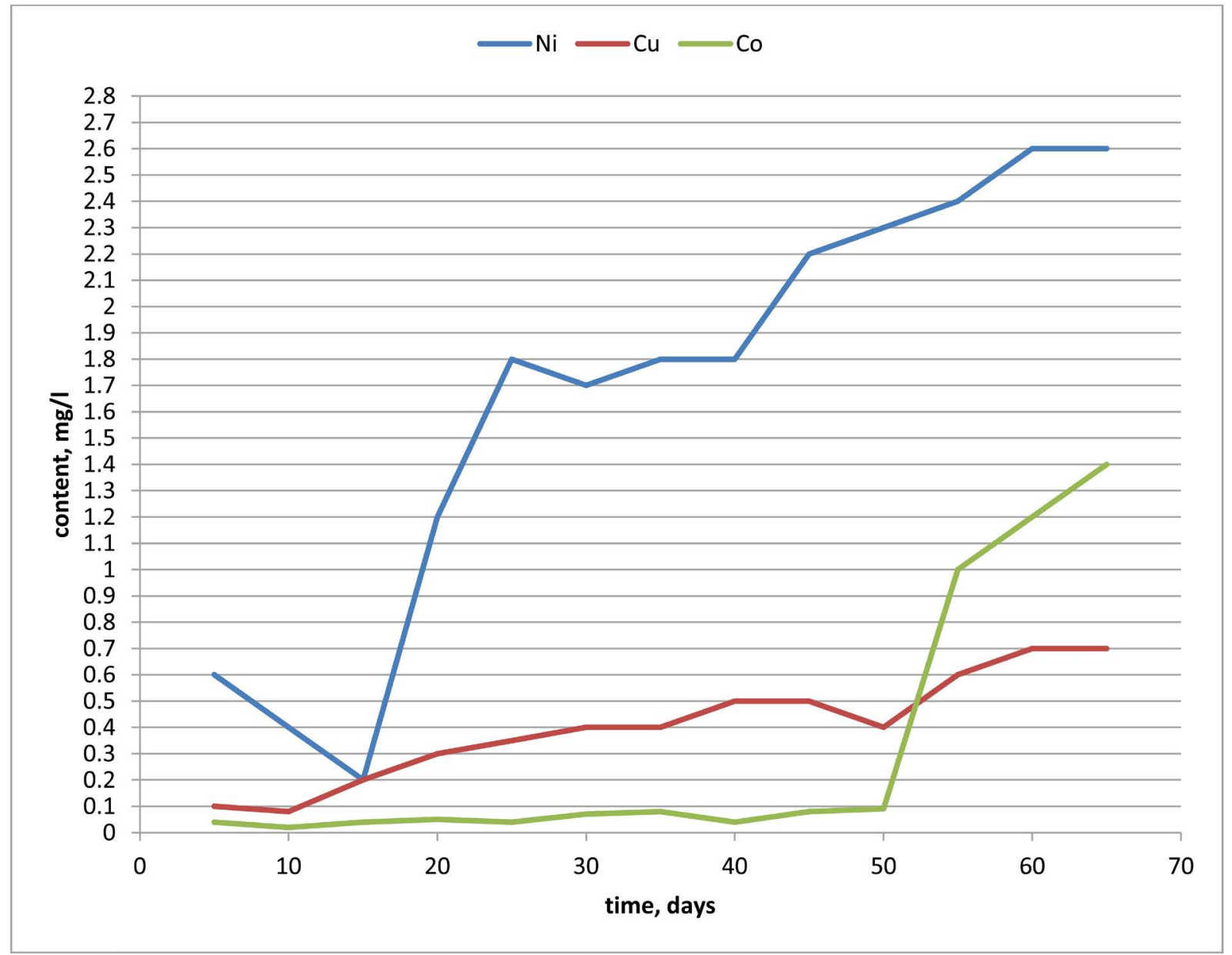

Figure 7. The concentration of $\mathrm{Ni}, \mathrm{Cu}$, Co in the solution on the surface of the material in the column.

of the degree of transition of the metal in solution on the thickness of the filtering layer is maintained: the thicker the layer, the less metal in the solution in the initial period and the inverse picture at the end of the experiment.

The geochemical phase analysis [5] [9] of the material at the beginning of the experiment and at its completion shows that during the experiment, most of the water-soluble forms of non-ferrous metals were removed by filtration of water through the array. However, one cannot speak of the full extraction of water-soluble forms from the massif. Geochemical analysis indicates the presence of all fractions in the material both at the beginning (Figure 8(a)) and at the end of the experimental period (Figure $8(b)$ ). Recalculation on the share of water-soluble forms of non-ferrous metal shows that during the whole period of the experiment, from the massif, in absolute values, on average $5 \%-12 \%$ more was extracted than there were water-soluble forms of non-ferrous metals in the initial stage of the experiment. Most likely, this fact can be explained with the oxidative transformation of compounds of non-ferrous metals during the process of hypergenesis in the period of the experiment than by the magnitude of the error of geochemical phase analysis. The geological natural process of hypergenesis in the presence of filtration does not stop, and even more than that, 


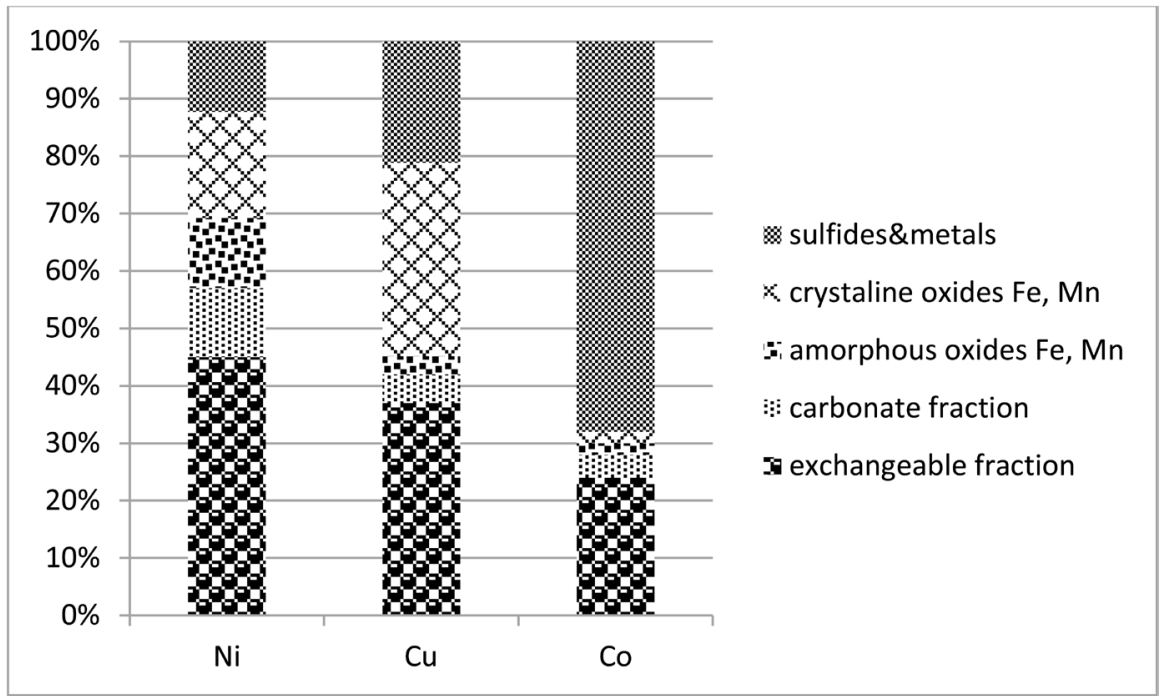

(a)

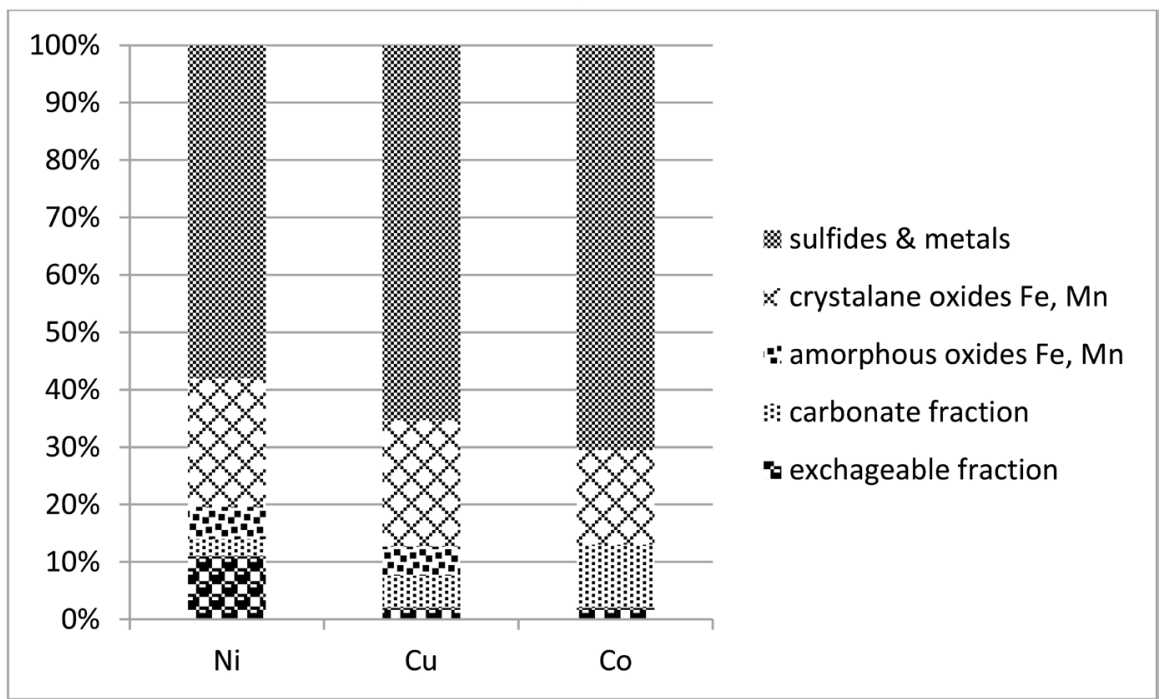

(b)

Figure 8. Geochemical phase analysis of non-ferrous metals at the beginning a) and at the end $b$ ) of the experiment.

it proceeds more intensively than when there is no access of oxygen to the massif.

Experimental studies with the upward movement of solutions in the array, at the water base, are aimed at carrying out a fundamental assessment of the technological applicability of directed concentration formation in the near-surface place of the massifs.

The development option for the natural site of the deposit can be formed according to the following schemes (Figure 9, Figure 10) using the hydrogeological resource of the subsoil. An array of the subsoil with localized mineralization, is subjected to explosive loosening to increase permeability. The subsoil section is drained to the lower level. Filling with the leach solution is carried out through the central wells from below. On the surface, a geochemical barrier or collection 


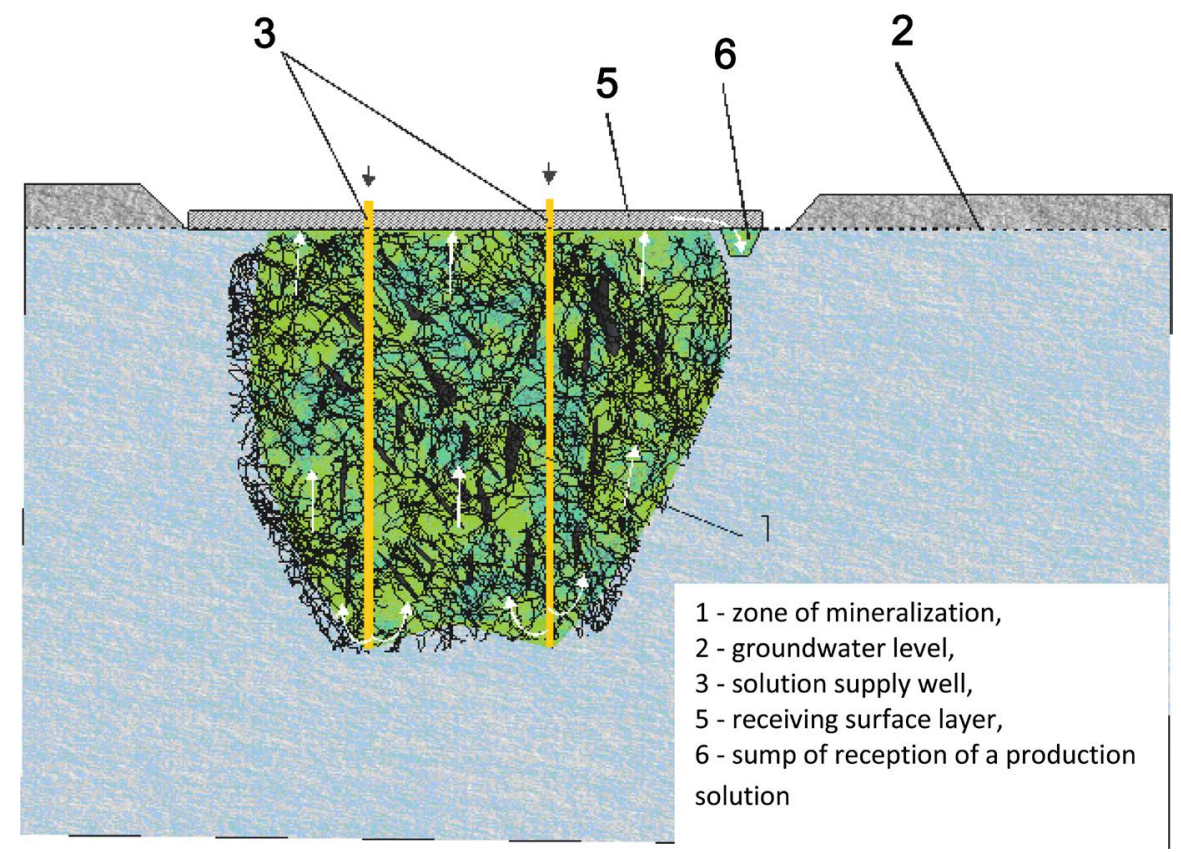

Figure 9. Technological scheme of deposit preparation on the situ.

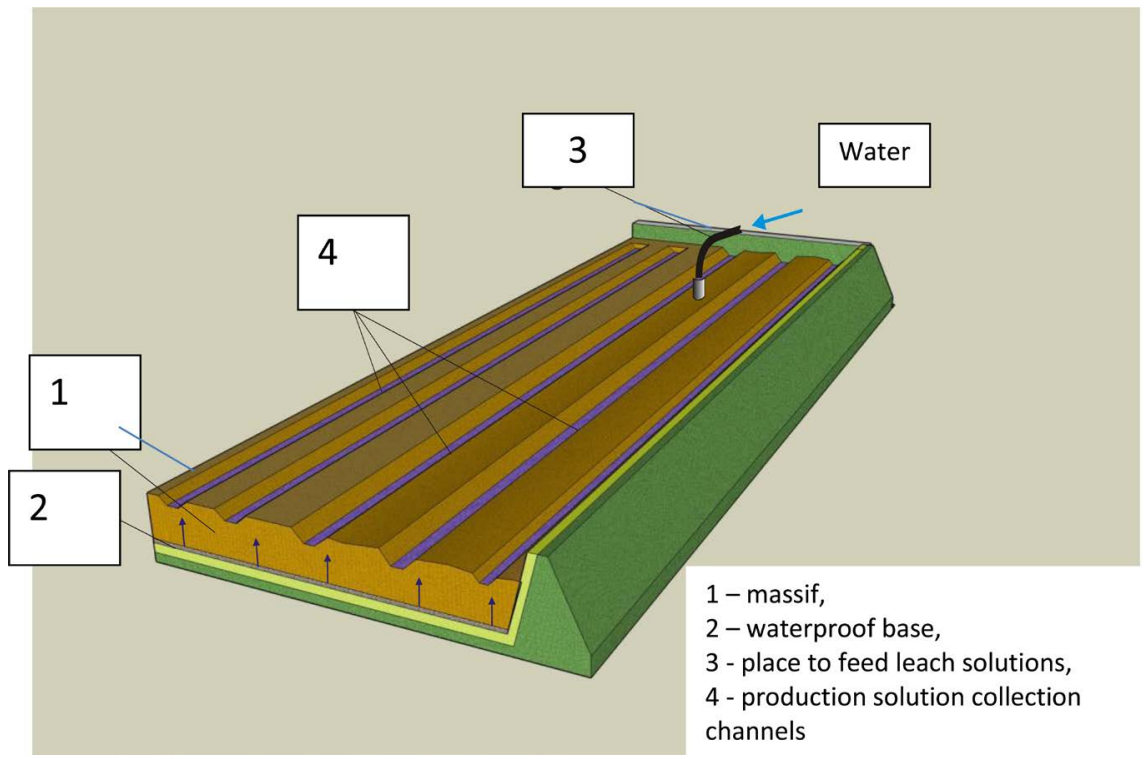

Figure 10. Technological scheme of preparation of tailings of enrichment.

of the production solution is formed. After that, the supply of solution is commensurate with the rate of capillary rise to the surface. Useful components are removed from the geochemical barrier after storage or removed from the solution under cyclic rotation conditions of the solution through the array.

For a technogenic object, for example tailings of flotation enrichment, the preparation scheme can be represented as follows (Figure 9). Wells pass from the surface to the bottom of the waste massif. Wells are distributed evenly over the entire area. Through the wells, the leaching reagent is fed into the lower zone of the massif. The solution leaches useful components and rises along the capil- 
laries of the massif to the surface. A geochemical barrier can be formed on the surface to precipitate useful components, or a production solution can be collected with its supply for extraction in each cycle.

\section{Conclusions}

An experiment carried out with water as a leaching agent for moving water-soluble compounds of non-ferrous metals in the massif of flotation tailings shows that the geological processes of hypergenesis allow the leaching by water to conduct a directed preliminary concentration of non-ferrous metals near the surface. This way allows the subsequent intensive extraction of useful components. To accumulate useful components in close proximity to the surface of the array, both physical barriers (evaporation) and geochemical (sorption) barriers can be used.

The non-ferrous metals are constantly present in the aqueous solution throughout the entire experiment. The concentration of the salts of non-ferrous metals in the solution is not the same at different levels of the massif. In the initial period of the experiment, the trend of decreasing all elements of non-ferrous metals in solution was from the bottom to up. In the final period of the experimental cycle, the concentration of non-ferrous metals in the upper zone exceeds the concentration of the lower zone by a factor of 2 - 4. The mass exchange process of hypergenesis carries out a gradual redeposition of the salts of non-ferrous metals from the bottom up in the direction of movement of aqueous solutions. The concentration of the zone of non-ferrous metals shifts in the direction of movement of aqueous solutions in the array. After 1.5 years of experiment, the concentration of water-soluble compounds of non-ferrous metals moved to the surface. Such results allow us to propose technological schemes for preparing the deposit for development. This approach to the development of deposits is suitable for natural deposits with a low sub-grade content of useful components in the massif. Also, this approach is applicable to technogenic objects, such as wastes of enrichment and warehouses of substandard ores. The basic schemes are given in the materials of this article.

\section{References}

[1] Kharitonova, M.Y., Mazko, N.A. and Mikhailov, A.G. (2013) Influence of the Time Factor on the Availability of Deposits of Nonferrous Metals. Resources Policy, № 38, 490-495. https://doi.org/10.1016/j.resourpol.2013.06.006

[2] Peshkov, A.A. and Matsko, N.A. (2004) Availability of Mineral Raw Materials. Nauka Press, Moscow, 280 p.

[3] Smirnov, V.S. (1976) Geology of Minerals. Nedra, Moscow, 688 p.

[4] Ma, Y., Feng, S., Zhan, H., Liu, X., Su, D., Kang, S. and Song, X. (2011) Water Infiltration in Layered Soils with Air Entrapment: Modified Green-Ampt Model and Experimental Validation. Journal of Hydrologic Engineering, 16, 628-638. https://doi.org/10.1061/(ASCE)HE.1943-5584.0000360

[5] Piatak, N.M., Seal II, R.R., Sanzolone, R.F., Lamothe, P.J. and Brown, Z.A., (2006) 
Preliminary Results of Sequential Extraction Experiments for Selenium on Mine Waste and Stream Sediments from Vermont, Maine, and New Zealand. U.S. Geological Survey Open-File Report 2006-1184, 21 p.

[6] Shokri, N. and Salvucci, G.D. (2011) Evaporation from Porous Media in the Presence of a Water Table. Vadose Zone Journal, 10, 1309-1318. https://doi.org/10.2136/vzj2011.0027

[7] Matsko, N.A. and Kharitonova, M.Yu. (2005) Mesoeconomic Approach to Assessing the Availability of Raw Materials in the Territory. Mining Information and Analytical Bulletin, Issue 10, 142-148.

[8] Peshkov, A.A., Matsko, N.A., Mikhailov, A.G. and Bragin, V.I. (2015) Prospects of Use of Megatechnologies for Development. Nanobiotechnologyin Energy, Environment and Electronics. In: Nicolini, C., Ed., Methods and Applications, Pan Stanford Publishing, Singapore, 197-213.

[9] Camm T.W. (1991) Simplified Cost Model for Prefeasibility Mineral Evaluation. Bureau of Mines Information Circular, United States, 35 p. 